\title{
Direct Observation of Anisotropy-Driven Formation of Amyloid Protein Core-Shell Structures in Real-time by Super-resolution Microscopy
}

Min Zhang ${ }^{1}$, Henrik Pinholt ${ }^{1}$, Xin Zhou ${ }^{2}$, Søren S.-R. Bohr ${ }^{1}$, Luca Banetta ${ }^{3}$, Alessio Zaccone ${ }^{4}$, Vito Foderà $^{2}$ * and Nikos S. Hatzakis, ${ }^{1,5 *}$

${ }^{1}$ Department of Chemistry, Faculty of Science, University of Copenhagen, Denmark

${ }^{2}$ Department of Pharmacy, Faculty of Health and Medical Sciences, University of Copenhagen, Denmark

${ }^{3}$ Department of Applied Science and Technology" at Politecnico di Torino in Turin ${ }^{4}$ Department of Physics, University of Milan, Italy

${ }^{5}$ NovoNordisk centre for Protein research, Faculty of Health and Medical Sciences University of Copenhagen Denmark

*corresponding authors: vito.fodera@sund.ku.dk (VF); hatzakis@chem.ku.dk (NSH) 


\begin{abstract}
Proteins misfolding and aggregation in the form of fibrils or amyloid containing spherulite-like structures, are involved in a spectrum of degenerative diseases. Current understanding of protein aggregation mechanism primarily relies on conventional spectrometric methods reporting the average growth rates and microscopy readouts of final structures, consequently masking the morphological and growth heterogeneity of the aggregates. Here we developed REal-time kinetics via binding and Photobleaching LOcalization Microscopy (REPLOM) super resolution method to observe directly and quantify the existence and abundance of diverse aggregation morphologies as well as the heterogeneous growth kinetics of each of them. Our results surprisingly revealed insulin aggregation is not exclusively isotropic, but it may also occur anisotropically. Combined with Machine learning we associated growth rates to specific morphological transitions and provided energy barriers and the energy landscape for each aggregation morphology. Our unifying framework of detection and analysis of spherulite growth can be extended to other protein systems and reveal their aggregation processes at single molecule level.
\end{abstract}


Protein misfolding and aggregation in the form of fibrils or amyloid containing spherulite-like structures are a hallmark of a number of devastating pathologies, such as Alzheimer's and Parkinson's disease ${ }^{1,2}$. Indeed, elongated protein aggregates named amyloid-like fibrils are found in such diseases and, in the last decades, deciphering the key steps of their formation has been the main focus of the amyloid community ${ }^{3,4}$. However, a "one-size-fits-all" approach for the description of amyloid forming systems cannot be used. Other amyloid-like species (named superstructures) may occur, exhibiting a significantly different $\beta$-sheet packing when compared to fibrils ${ }^{5,6}$. Among others, amyloid-like spherical aggregates, named spherulites, ranging from few micrometers to millimeters in diameter can be formed both in vivo and in $v$ itro $^{6-8}$. These aggregates are characterized by a fascinating core-shell morphology and seems to be the result of a general self-assembly pattern, which is common for metal alloys ${ }^{9}$, minerals ${ }^{10}$, and polymers ${ }^{11,12}$. Notwithstanding protein amyloid spherulites are hallmark for diseases states ${ }^{1,2}$ and they may also represent opportunity as advanced materials in drug delivery ${ }^{13}$, our understanding of the mechanisms of formation and growth of spherulite is quite limited ${ }^{14,15}$.

Current models for the mechanism of protein spherulites formation primarily rely on spectrometric evidence reporting average growth rates ${ }^{14,16}$ and observation of the final structures via microscopy techniques ${ }^{7,17}$. This evidence has brought to the hypothesis that spherulites are core-shell structures, in which fibril-like filaments isotropically and radially grow on a dense core ${ }^{7,18,}$ following a multifractal pattern driven by electrostatic interactions ${ }^{19}$. The complexity of such scenario is further enhanced by the fact that in vitro amyloid spherulites co-exist with fibrils ${ }^{19,20}$. Such high heterogeneity of the aggregate populations within the same self-assembly reaction represents a further obstacle, preventing the correct evaluation of the multiple and concurrent pathways. Indeed, while bulk methods guarantee a rapid screening of the overall propensity of proteins to form amyloid structures ${ }^{6}$, they provide limited information on the aggregation kinetics of the individual species, either fibrils or spherulites, averaging the effect of morphological heterogeneity of the aggregate population. Consequently, novel methods for real time and direct observation of single aggregate growth to develop and inform models accounting for the heterogeneous growth are highly desirable. 
Here we initially combined astigmatism-based 3D direct stochastic optical reconstruction microscopy (dSTORM $)^{21}$, spinning disk confocal microscopy ${ }^{22}$ and scanning electron microscopy (SEM) to directly observe the formation and kinetics of individual protein amyloid structures using human insulin (HI) as a model system. Our results allowed us to differentiate among the different species in solution and decipher the nature, morphologies and abundance of individual spherulites at different growth stages. Surprisingly, HI spherulite growth is not exclusively isotropic, but it may also occur anisotropically. We developed a method for the detection of REal-time kinetics via binding and Photobleaching LOcalization Microscopy (REPLOM), to attain real-time video of spherulites growth process, reconstruct the super-resolution image of the spherulites and their growth kinetics. Using home-made software based on Euclidian minimum spanning tree and machine learning clustering ${ }^{23-26}$, we quantitatively associated growth rates to specific morphological transitions during the growth, eventually extracting detailed energy barriers and thus the energy landscape for each morphology type of the aggregation. These findings underscore how conclusions solely based on bulk kinetics data may overlook the complexity and the heterogeneity of the aggregation process. We anticipate the framework presented here to serve as a unique generic methodology for the simultaneous detection and analysis of multiple species within a single protein aggregation reaction, intimately connecting kinetics, morphological transitions and structure. 

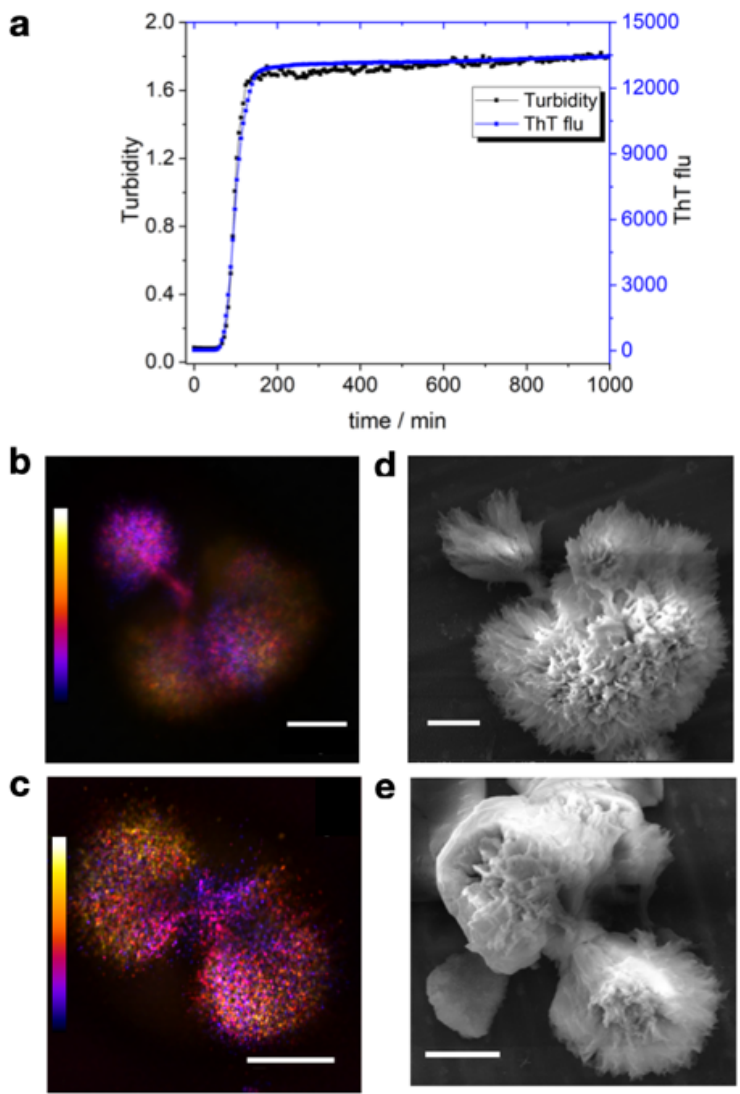

Figure 1. Bulk kinetics and structure of anisotropically grown HI spherulites of two distinct growth-morphologies. a) ThT fluorescence and turbidity kinetics showing that the aggregation process is complete in $\sim 3 \mathrm{~h}$ at $60{ }^{\circ} \mathrm{C}$ and that turbidity and fluorescence provide identical results. b) and d) spherulites with two asymmetric sides captured by spinning disk confocal microscopy and SEM respectively. c) and e) spherulites with symmetric two-side-structures captured by spinning disk confocal microscopy and SEM, respectively. Data in b) and c) were acquired for a sample with incubation time of $16 \mathrm{~h}$ at $60{ }^{\circ} \mathrm{C}$. Data in d) and e) are from a sample with incubation time of $4 \mathrm{~h}$ at $60^{\circ} \mathrm{C}$. Color scale is from $-14.04 \mu \mathrm{m}$ to $14.04 \mu \mathrm{m}$ in c) and $-8.46 \mu \mathrm{m}$ to $8.46 \mu \mathrm{m}$ in d). Scale bars are $10 \mu \mathrm{m}$. All the samples were covalent labelled with Alexa Fluor 647.

\section{Results}

We thermally induced insulin amyloid aggregation using an established protocol ${ }^{27}$ and followed the bulk kinetics via detecting the fluorescence of the amyloid-sensitive dye Thioflavin T (ThT) and the turbidity signal (Fig. 1a). The kinetics traces show the classical three-step profile, with the reaction reaching a completion already after 3-4 hours. Moreover, turbidity and ThT signal perfectly overlap, suggesting that the aggregation reaction is entirely of amyloid-like origin ${ }^{28}$. However, standard analysis of the bulk ThT signal is unable to provide information on the morphological transition occurring during the reaction. To this aim, we used spinning disk microscopy and SEM to detect the morphology of spherulites at different growth stages (see Supplementary Fig. 1). Interestingly, we detect both spherulites with asymmetrically grown (Fig. 
$1 \mathrm{~b}$ and $1 \mathrm{~d}$ ) and symmetrically grown (Fig. $1 \mathrm{c}$ and $1 \mathrm{e}$ ) lobes (see 3D videos of Fig. $1 \mathrm{~b}$ and $1 \mathrm{c}$ in supplementary movies 1 and 2). We confirmed asymmetric growth is not an artefact of substrate depletion as spherulites with asymmetric lobes are already formed after 2 hours of incubation (Fig. 2) This suggests the occurrence of growth with multiple rates within a single sample (Supplementary Fig. 1b), which may be masked in bulk kinetics. Moreover, our data indicate the possibility of a growth not entirely occurring isotropically from the central core, but rather having initially some preferential direction.

a

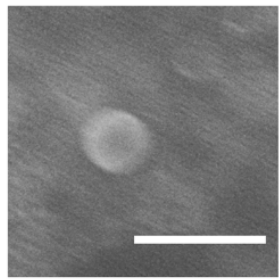

C

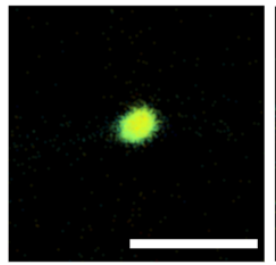

d

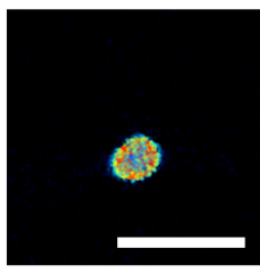

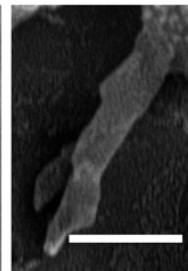
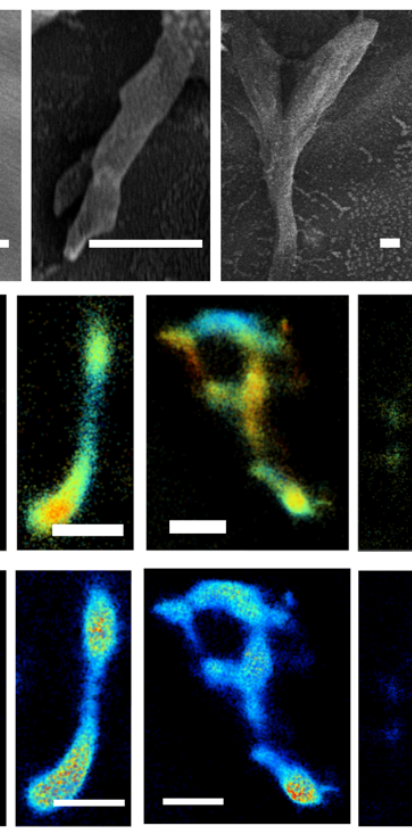
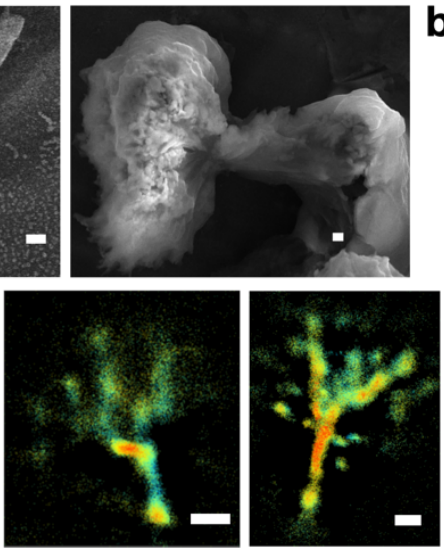
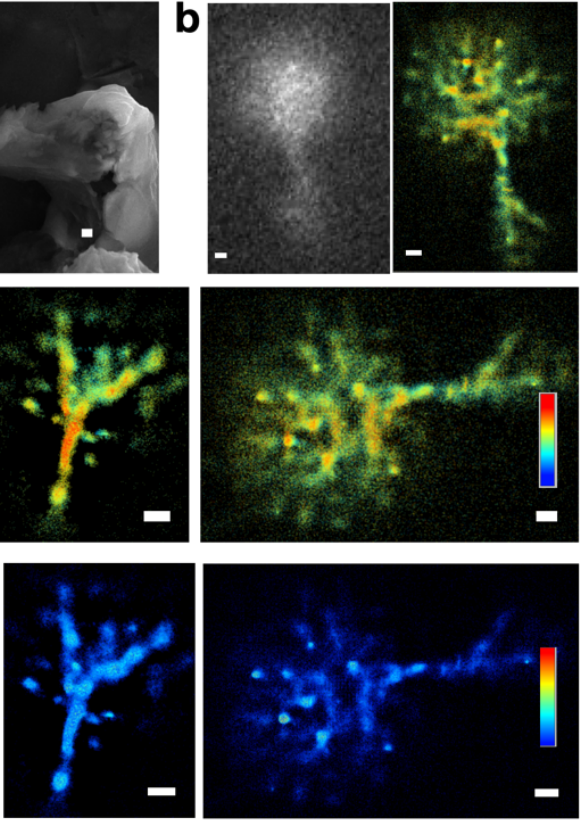

Figure 2. SEM and super-resolution 3D dSTORM images capturing SNAPshots of the morphological transition during anisotropic growth of different $\mathrm{HI}$ aggregates. a) SEM images capturing the multiple different steps of the aggregate growth at incubation time from 0.5 hour to 2 hours for samples incubated at $60{ }^{\circ} \mathrm{C}$. b) 3D dSTORM image of anisotropic spherulite (right) and its corresponding diffraction limited fluorescence image (left). c) and d) 3D dSTORM images at different steps of the aggregate growth at incubation time from 0.5 hour to 2 hours and incubation temperature at $60{ }^{\circ} \mathrm{C}$. $\mathrm{c}$ ) depth plots, pseudocolor scale is from $-500 \mathrm{~nm}$ to $500 \mathrm{~nm}, \mathrm{~d}$ ) density plots, pseudocolor scale corresponds to neighbour localizations: the density of neighbouring events within an $100 \mathrm{~nm}$ radius sphere from localisation. Pseudocolor scale ranges from 0 to 1000 for the first image on left and 0-400 for the rest. Scale bars are $1 \mathrm{um}$.

To further confirm the asymmetric and the directionality of the growth, we combined the insights from SEM and 3D dSTORM that offer direct and high-resolution recordings of the early events preceding the full formation of a spherulite structure. Figure 2a shows the SEM images of the sample at different incubation times between 0.5 to 2 hours. Spherical-like protein condensates of approximately $200 \mathrm{~nm}$ in diameter are firstly formed after 0.5 hours. Interestingly, these further grow following a linear pattern. This suggests that protein spherulite growth may proceed 
towards a preferential direction, and not only isotropically in space as originally postulated 7,19 (Fig. 2a). After this linear growth, branching phenomena occur on the two ends of the aggregates, activating the growth of the spherulite lobes (Fig. 2).

3D dSTORM allowed us to extend beyond diffraction limited imaging, that may mask spherulite shape and growth directionality (Fig. 2b), observe directly similar growth pathways and confirm anisotropic spherulite growth (see Methods). It also showed that this is not an artefact from sample drying for SEM imaging ${ }^{7}$ (Fig. 2c). Consistent with the data in Fig. 1, the growth of the two lobes may proceed with different reaction rates resulting in unequal lobe size at a specific point in time (Fig. 2c, right panel). It is worth noting that heterogenous growth rate results in diverse size and morphology of aggregates at the same incubation time (Supplementary Fig. 1c and 1d). The density plots offered by 3D dSTORM, clearly show that the core has much higher density than the branching part, consistent with previous suggestions on the existence of a low-density corona in the spherulites structures ${ }^{7,18}$ (Fig. 2d). The formation of the high-density cores appears to represent the nucleation point and the subsequent linear-like elongation and branching of slender threadlike fibrils resemble crystalline growth ${ }^{29,30}$. More specifically, our data indicate an initial process driven by liquid-to-solid transition as recently proposed for a number of protein systems ${ }^{31}$, while the further growth is determined by a tight fibril packing, which forces the biomolecular assembly to occur anisotropically and along one specific direction. The latter is in contrast to isotropic spherulite growth, where the process occurs via the formation of a radiating array of fiber crystallites, but it is observed in the case of crystalline-coil block copolymer spherulites $^{32}$. The origin of such anisotropy might be due to the occurrence of secondary and heterogeneous nucleation at the aggregate surface ${ }^{33}$, with different binding efficiency depending on the aggregate areas, but deciphering this with additional data falls out of the scope of this work.

The section of the early linear aggregates increases as a function of the time (Supplementary Table 1). This indicates that the growth is not limited to an end-to-end attachment to the linear aggregate and lateral aggregation can also take place. The central early linear structures with diameter $400 \pm 100 \mathrm{~nm}$ (see Supplementary Table 1) successively branches to form radially oriented amyloid fiber-like structure; the further away from the core the more increased the 
bioRxiv preprint doi: https://doi.org/10.1101/2021.08.20.457097; this version posted August 20, 2021. The copyright holder for this preprint (which was not certified by peer review) is the author/funder, who has granted bioRxiv a license to display the preprint in perpetuity. It is made available under aCC-BY-NC-ND 4.0 International license.

branching frequency, yielding more space-filling patterns. The dimensions of a corona-like structure expand from $\sim 2 \mu \mathrm{m}$ to $>20 \mu \mathrm{m}$, as shown in Fig. $2 \mathrm{c}$.

a
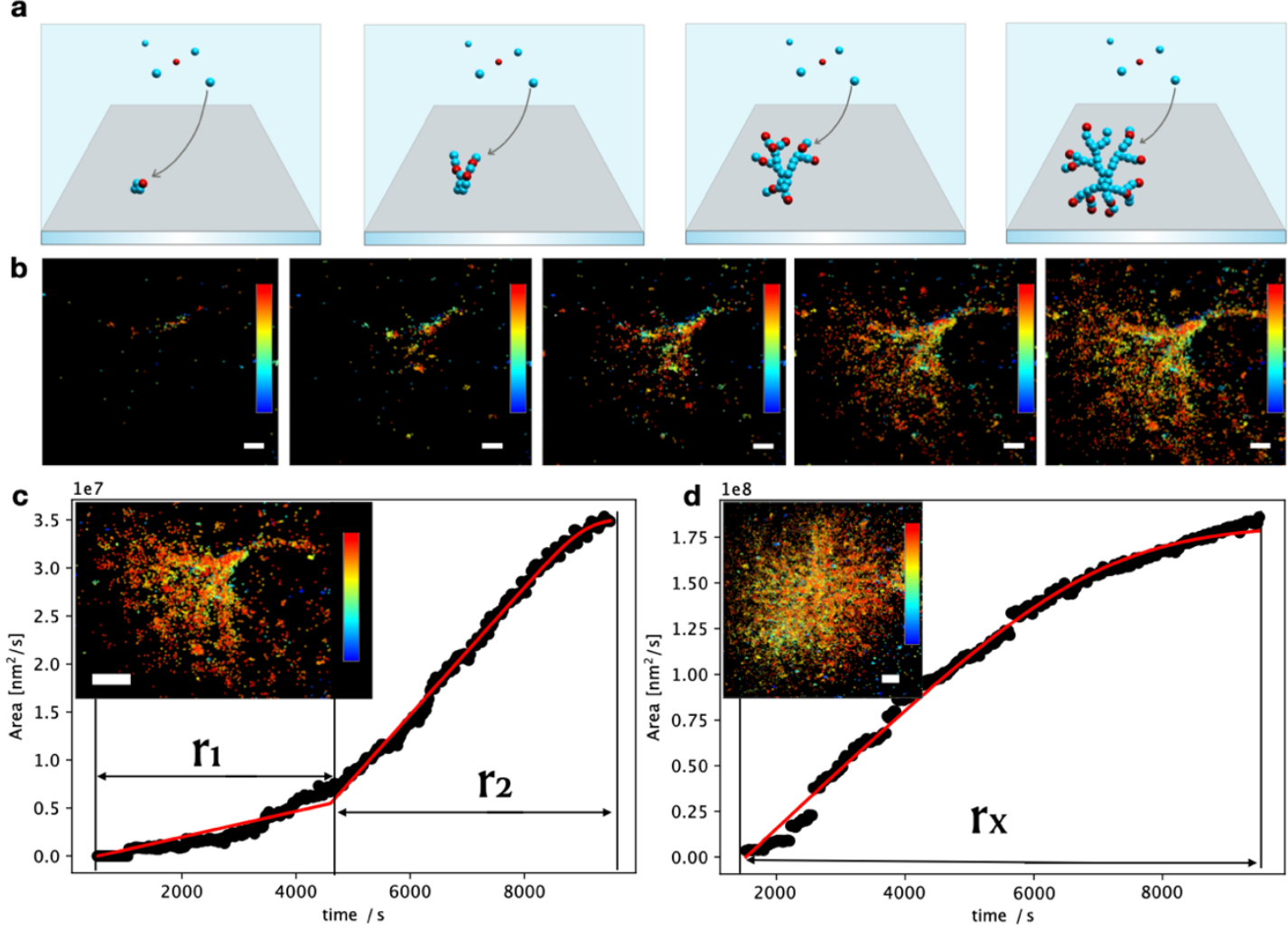

Figure 3. Direct real time observation of $\mathrm{HI}$ aggregates growth by REPLOM (real-time kinetics via binding and photobleaching localization microscopy). a) Cartoon representation of REPLOM: Initially, the fluorescent signal from the small fluorescently labelled protein condensates is detected followed by the chromophore photobleaching. As the growth progresses, labelled insulins from solution will bind extending the aggregate dimensions. Each binding event will result to a diffraction limited spot, the coordinates of which are accurately extracted, before it photobleaches by the intense laser. Parallelised recordings of the spatially distinct binding of multiple individual emitters reveal the temporal morphology development of several aggregates (Red is Alexa Fluor 647 labelled $\mathrm{HI}$ is on fluorescent state, and blue is un-labelled insulin or Alexa Fluor 647 labelled $\mathrm{HI}$ in a dark/photobleached state). b) Direct real time observation of temporal development of anisotropic growth at $t=1900$ time intervals. Scale bars: $1 \mu \mathrm{m}$. Color bars: $0 \mathrm{~s}$ (blue) to $1900 \mathrm{~s}, 3800 \mathrm{~s}, 5700 \mathrm{~s}, 7600 \mathrm{~s}$ and $9500 \mathrm{~s}$ (red, from left to right respectively). c) and d) Growth curves of anisotropic spherulite (c) and isotropic spherulite (d). For anisotropic spherulite, the curve contains two parts roughly correlating with the formation of the core/linear part and branching part (see method REPLOM section) . Isotropic spherulite growth is linear followed by saturation. Inset: the corresponding HI spherulite obtained by RE-PLOM. Scale bars: $2 \mu \mathrm{m}$. The color bar ranges from $0 \mathrm{~s}$ (blue) to $9500 \mathrm{~s}$ (red). See SI for the movie.

Data in Fig. 1-2 offer a novel and unique picture of the pronounced diversity in structures, which one can obtain within a single aggregation reaction. Together with this, our findings point out the occurrence of a heterogeneous distribution of growth rates that may dramatically affect the course of the kinetics and the final morphology of the aggregates. This highlights once more, that bulk methods ${ }^{19}$ dramatically undermine such morphologically diverse growth and their respective kinetics, potentially leading to erroneous evaluation of the rate constants. Diffraction limited microscopy or super resolution microscopy, on the other hand, can only show the 
morphologies of aggregates, making hard to extract rate constants and thermodynamic parameters for the process.

To overcome this limitation and quantitatively measure the growth rates at single aggregate level and simultaneously monitor the morphological development of the structure, we developed a new super resolution experimental method, based on single molecule localization microscopy. We name it REal-time kinetics via binding and Photobleaching LOcalization Microscopy (REPLOM), as it allows for the first time to directly image the morphological development of each individual spherulite in real-time with super resolution and, simultaneously access the kinetics traces for the thermodynamics analysis of the process. To perform REPLOM, HI monomers were covalently labelled with Alexa Fluor 647 NHS Ester, the optimal ratio of labelled to unlabelled HI monomer was 1:10000 (see Supplementary Information for experimental details). Fig. 3a shows how REPLOM works: initially, only small protein condensates, i.e. cores, are formed and bind to the poly-L-lysine covered surface. The spatial localisation of each of the fluorophores is accurately detected prior to their photobleaching ${ }^{34,35}$. As the growth progresses, additional $\mathrm{HI}$ monomers from the solution binds to the core, extending the aggregate dimensions. Each labelled insulin binding will result in a diffraction limited spot, the precise localisation of which, can be accurately extracted similarly to PALM methodologies ${ }^{36}$ (see Methods and Supplementary Fig. 8 for resolution of the method, supplementary movie 3-4). Optimised imaging settings and absence of imaging buffer ensures the rapid chromophore bleaching after binding (see Methods and Supplementary Fig. 7) Parallelised recordings of the spatially distinct binding of multiple individual human insulin loaded emitters, allow the real time direct observation of the temporal morphology development of each of aggregates (see Fig. 3b and Supplementary movies, 3-4). The methodology is similar to Binding Activation Localisation Microscopy (BALM) ${ }^{37}$ measuring existing structures, but offers in addition, the real time direct observation of the growing process. Consequently, the geometry and the morphological development of each aggregate is observed directly with sub-diffraction resolution, offering extraction of each particle's growth kinetics.

Consistent with the 3D dSTORM data, direct observation of $\mathrm{HI}$ spherulite growth by RE-PLOM, confirmed that $\mathrm{HI}$ spherulites can grow both anisotropically and isotropically (Fig. 3). To extract growth rate kinetics for each individual aggregate, we identified points belonging to the growing aggregate with an approximate Euclidean Minimum Spanning tree segmentation ${ }^{38}$ and estimated 
the area using a gaussian mixture model, based on hierarchical clustering in Fig. $3 c$ and $3 d$ (see Supplementary information for the details) ${ }^{23-26}$. For isotropic morphologies a single linear growth rate is observed $\left(r_{x}\right)$, while for anisotropic morphologies the growth curve consists of two rate components ( $r_{1}$ and $r_{2}$ ), as shown in Fig. 3c and 3d, and Supplementary Fig. 9. $r_{1}$ corresponds to the initial linear core part and $r_{2}$ to the branching part, and they are best fit with a reaction limited, linear growth and a diffusion limited, sigmoidal growth, respectively ${ }^{15,16,39-41}$ (see Methods and supplementary movies 3-8). Consequently, the growth rates $\left(r_{1}, r_{2}\right.$ and $\left.r_{x}\right)$ for each individual aggregate are extracted. Growth readouts of individual geometrically distinct morphologies allows to go beyond the standard analysis of sigmoidal curves which does not yield information on, or discriminating between, the temporal developments for each morphology. REPLOM revealed anisotropic growth to operate via a two-step process imposed by the geometry of the growth, a pattern masked in current super resolution and bulk readouts.
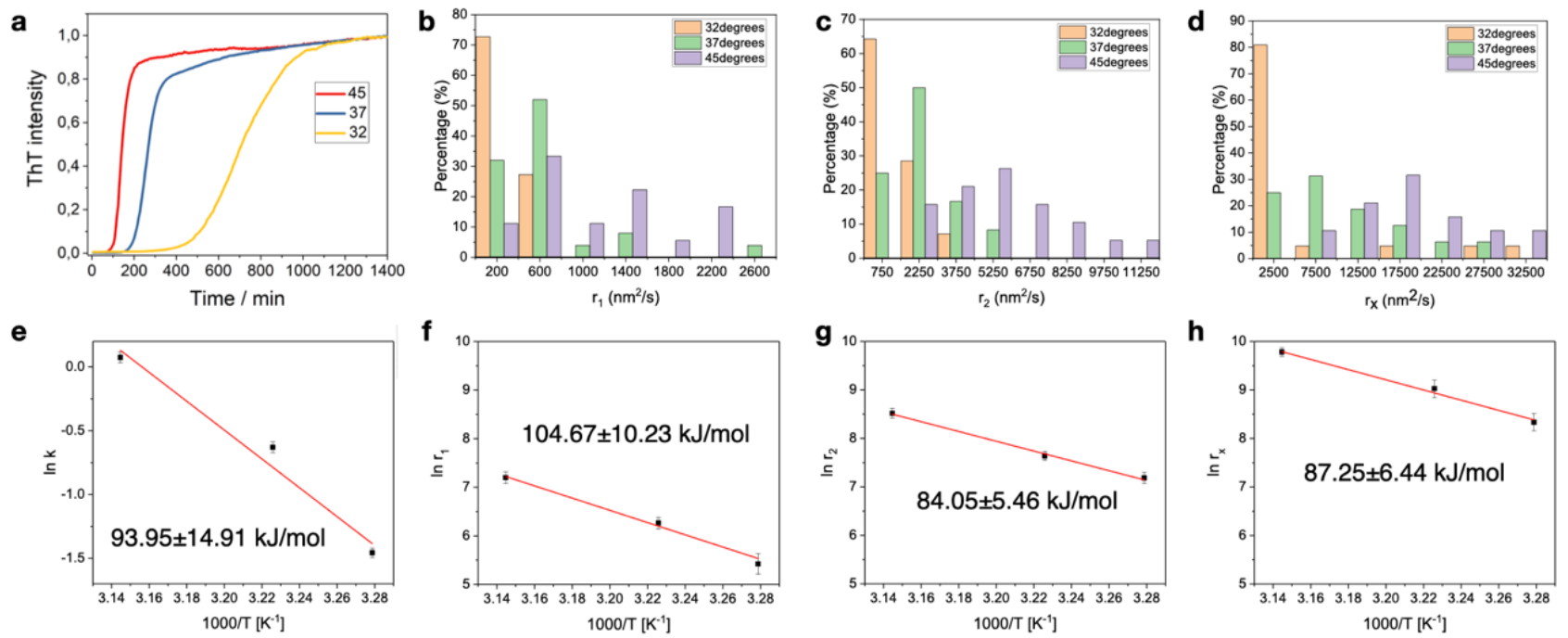

Figure 4 Kinetic and thermodynamic characterisation of insulin aggregation. a) Normalized bulk ThT fluorescence kinetics on with incubation temperatures of 45, 37 and 32 degrees respectively b) and c) REPLOM extracted rate distribution of anisotropic aggregates at the three different incubation temperatures: (b) the linear part and (c) the branching part. d) Rate distribution of isotropic aggregates at the three different incubation temperatures. e-h) Arrenhius plots for spherulites as obtained from bulk experiments (e), and REPLOM (f-h). The formation of linear ( $f$ ) and branches (g) parts of anisotropic spherulites and the formation of isotropic spherulites (h).

The real time single particle readout of REPLOM allowed kinetic analysis of the temperature dependence of growth for each type of spherulites morphology and, consequently, extraction of the activation energy barriers for both spherulite morphologies and growth phase. To do this $\mathrm{HI}$ aggregate formation was induced at three different temperatures: $45^{\circ} \mathrm{C}, 37^{\circ} \mathrm{C}$ and $32{ }^{\circ} \mathrm{C}$. The ThT fluorescence measurement at the three temperatures presenting average growth kinetics are shown in Fig. 4a (see methods). The rate distributions at the three temperatures for each 
type of morphology growth are shown in Fig. 4 b, $c$ and $d(N \sim 20$, see also Supplementary Fig. 10). As expected, the linear parts $r_{1}$ (Fig. 4b) and branches parts $r_{2}$ (Fig. 4c) as well as the isotropic growth rate $r_{x}$ (Fig. $4 d$ ), grew faster by increasing the incubation temperature. Data do not present a pronounced curvature, and this may be due to the narrow temperature range investigated here, in agreement with earlier studies ${ }^{42}$. This would suggest that the differences in heat capacity between the soluble states of the proteins and the transition states for aggregation are small ${ }^{42}$. Using Arrhenius equation ${ }^{42}$ (Fig. 4e-4h), the activation energy of each of the isotropic or anisotropic morphological growths and the respective linear or branching part of individual aggregate was extracted. For the linear part of anisotropic spherulites, the activation energy is $104.67 \pm 10.23 \mathrm{~kJ} / \mathrm{mol}$ (Fig. 4f), while $84.05 \pm 5.46 \mathrm{~kJ} / \mathrm{mol}$ for the branched part (Fig. 4g), and $87.25 \pm 6.44 \mathrm{~kJ} / \mathrm{mol}$ when spherulites grow isotropically (Fig. 4h). Activation energy extracted from the bulk kinetics in Fig. 4e $(93.95 \pm 14.91 \mathrm{~kJ} / \mathrm{mol})$, is consistent with data or bovine insulin fibril formation of $\sim 100 \mathrm{~kJ} / \mathrm{mol}^{42}$. The determined thermodynamic values are rather similar across morphologies, indicating fundamentally similar mechanisms ${ }^{43}$. These data together indicate how the pronounced heterogeneity of growth mechanisms and structures within the aggregation ensemble leads to a heterogeneity of the activation barriers. We indeed highlight that spherulite growth may proceed both isotropically and anistropically, the latter presenting a two-step process imposed by the geometry of the growth and characterized by two activation energies, which are markedly different compared to the one obtained by bulk kinetics or to the one for insulin fibrils ${ }^{42}$. 

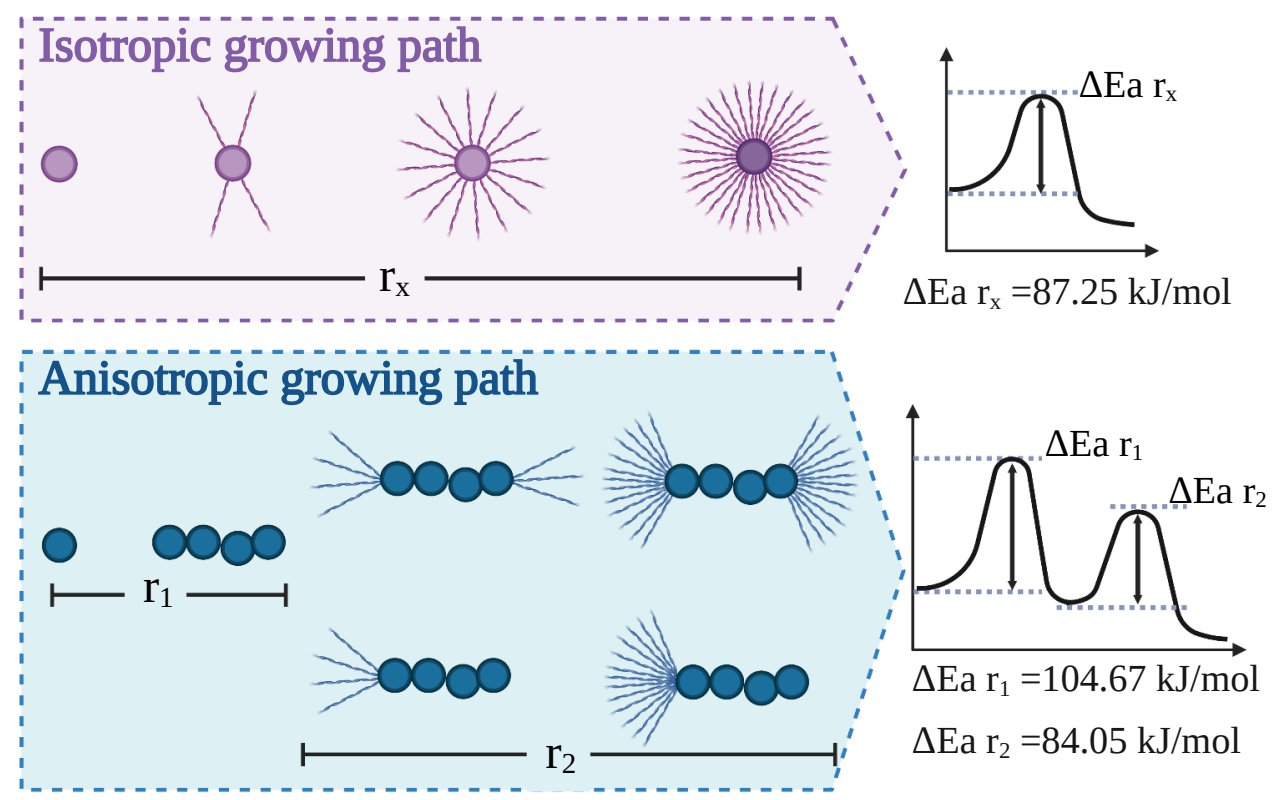

Figure 5. Cartoon representation of the diverse pathways of insulin aggregation and their respective energy barriers. Top : Isotropic spherulite growth, where fibril-like filaments isotropically and radially grow on a dense core. Process is characterised by a single activation energy of $\sim 87 \mathrm{Kj} / \mathrm{mol}$. Bottom: anisotropic growth, where the dense core is growing linearly before it successively branches to form radially oriented amyloid fiber-like structures. The further the branching from the core the more increased the branching frequency, yielding more space-filling pattern. Process is a two-step imposed by the geometry of the growth and characterized by two activation energies of 104 and $84 \mathrm{Kj} / \mathrm{mol}$ for the linear and branching part respectively.

In summary, we report a novel experimental approach allowing for a real-time detection of super resolution images during protein aggregation kinetics. The REPLOM method allows the direct observation of the self-assembly kinetics at the level of single aggregates, quantifying the heterogeneity of aggregates and their growth mechanisms, otherwise masked in experiments employing current methodologies. Our combined results revealed that the growth of amyloid core-shell structures named spherulites may proceed not only via an isotropic growth of the structure, but also following a multistep pathway characterized by an initial pronounced anisotropic behaviour. Such a variability in growth within the same aggregation reaction determines a spectrum of aggregation kinetics traces, which are quantitatively detected by our method, allowing one to extract the thermodynamic parameters for each of the aggregation subsets.

Our framework may contribute to a more comprehensive representation of the generalized energy landscape of proteins, via providing more quantitative information on the formation of each of the myriad of occurring aggregated structures. The method is easily translatable to more medically- relevant proteins, such as alpha-synuclein or A-beta peptide. In those cases, our 
approach may provide unprecedented hitherto information on transient intermediate species, which are nowadays recognized as the cause of the disease progression, both in terms of energetics and morphology. 


\section{Methods-Optical microscopy}

\section{Spinning Disk Microscopy.}

The 3D images of grown spherulites were taken by a SpinSR10-spinning disk confocal super resolution microscope (Olympus) using a silicone oil-immersion 100x objective (UPLSAPO100XS, $\mathrm{NA}=1.35$, Olympus). The Alexa Fluor 647-labeled $\mathrm{HI}$ spherulites were excited with a $640 \mathrm{~nm}$ laser (OBIS COHERENT). The exposure time was $50 \mathrm{~ms}$ and the $z$ step length was $0.36 \mu \mathrm{m}$.

\section{Scanning Electron Microscopy (SEM).}

SEM images of spherulites were taken by using a Quanta FEG 200 ESEM microscope.

\section{Cross Polarized Microscopy.}

Images were collected using a 10x objective and crossed polarised which enabled spherulites to show the characteristic Maltese cross (Zeiss Axioplan Optical Microscope, Carl Zeiss).

\section{Super resolution Imaging}

Super resolution imaging was attained on an inverted Total Internal Reflecion microscope (TIRF) (Olympus IX-83) with a 100x oil immersion objective (UAPON 100XOTIRF, NA=1.49, Olympus) Alexa Fluor 647 was excited by a $640 \mathrm{~nm}$ solid state laser line (Olympus) and reflected to a quad band filter cube (dichroic mirrors ZT640rdc, ZT488rdc and ZT532rdc for splitting and with singleband bandpass filters FF02-482/18-25, FF01-532/3-25 and FF01-640/14-25). Signal was detected by an EMCCD camera (imagEM X2, Hamamatsu)

3D direct Stochastic Optical Reconstruction Microscopy (3D dSTORM) and image analysis.

3D dSTORM imaging was achieved by installing a cylindrical lens ( $f=500 \mathrm{~mm}$ ) in the emission pathway of (TIRF) to introduce the astigmatism of point spread function (PSF) ${ }^{21}$. All the dSTORM imaging experiments were performed at room temperature $\left(21^{\circ} \mathrm{C}\right)$. The exposure time was 30 ms and 10000 frames for each movie.

To extract $\mathrm{z}$ information from the widths of single molecule images, we generated a calibration curve of PSF width in the lateral plane ( $W x$ and $W y$ ) as a function of height by measuring Atto 655-labeled liposomes using TIRF with a step size of $10 \mathrm{~nm}$ and exposure time of $30 \mathrm{~ms}$ (Figure S3).

The $\mathrm{HI}$ aggregates which were incubated in a block heater for 0.5 hours to 2 hours at $60^{\circ} \mathrm{C}$. At the desired time they were added to the poly-L-Lysine treated microscope chamber ${ }^{44}$ and incubated for $10 \mathrm{~min}$ at room temperature to ensure immobilization. Extra sample was washed away with MilliQ water. Imaging buffer containing $50 \mathrm{mM}$ Tris, $10 \mathrm{mM} \mathrm{NaCl}, 10 \%$ (w/v) glucose, $0.5 \mathrm{mg} / \mathrm{mL}$ glucose oxidase, $40 \mu \mathrm{g} / \mathrm{mL}$ catalase and $0.1 \mathrm{M} \mathrm{MEA}{ }^{45}$ was flushed into the chamber for dSTORM imaging. All measurements were carried out at room temperature. The optimal ratio of labeled to unlabeled insulin that provided reliable signal without affecting the aggregation process or compromising resolution was 1 to 60,000 . This is quite different from earlier dSTORM 
imaging of fibrils using a ratio of $1 / 20^{46}$ because of the much higher $3 D$ density of spherulites that prevent reliable super resolution imaging at high labeling ratios.

The 3D dSTORM data was analyzed by ThunderSTORM ${ }^{47}$. The $z$ information of individual localizations was extracted based on the calibration curves (calculated by ThunderSTORM, shown in Fig S3). The detected localizations were further filtered according to their intensity and drift correction, in order to remove some possible false positive or poor quality detections. 3D superresolution images were visualized with ViSP software ${ }^{48}$.

\section{REal-time kinetic via Photobleaching LOcalization Microscopy (RE-PLOM). Preparation of $\mathrm{HL}$ aggregates and imaging}

The solution containing $5 \mathrm{mg} / \mathrm{mL} \mathrm{HI}$ monomer was first incubated in a block heater to skip the lag phase. The optimal pre-incubation time for spherulite formation on the microscope surface was found to be $\sim 8$ hours for $45^{\circ} \mathrm{C}, 20$ hours for $37^{\circ} \mathrm{C}$ and 75 hours for $32{ }^{\circ} \mathrm{C}$, respectively. Then they were transferred to poly-L-lysine coated glass slide chambers and covered by a lip to prevent solvent evaporation during imaging.

RE-PLOM was performed on the same TIRF microscope setup as the 3D STORM without the cylindrical lens. Alexa Fluor 647 labeled $\mathrm{HI}$ was excited by $640 \mathrm{~nm}$ solid state laser lines (Olympus). Imagign was performed with an exposure time of $30 \mathrm{~ms}$ followed by a waiting time for each frame of 30 seconds so as to capture in real time the slow kinetics of spherulite formation. All image acquisition was performed at the same incubation temperatures as in the block heater. The incubation temperatures during the imaging processes were achieved by a heating unit 2000 (PECON).

The data was analyzed by ThunderSTORM. Some possible false positive or poor-quality detections were removed by intensity filter. Fig S7 shows the comparison of images prior to and after drift correction. The reconstructed images with time series were obtained by ViSP 48 software. The resolution of RE-PLOM was determined by the FWHM of single spot's intensity (Figure S8) as recently described ${ }^{49}$ using an adapted version of previously published software ${ }^{35,50}$. Briefly, using our subpixel resolution software, we were able to extract multiple (91) single spots (see Fig. S8) and align all to the same center. Fitting a two-dimensional gaussian to the resulting stacked clusters allowed the reliable extraction of FWHM used to determine the obtained resolution. Using a maximum likelihood fitting scheme avoided potential bias from data binning.

The lifetime of fluorophores in RE-PLOM was evaluated by checking the duration time of fluorescent state before they were photobleached. We checked 1885 individual Alexa Fluor 647 fluorophores and found they were photobleached very fast without imaging buffer (Figure S7). The lifetime is about $0.7845 \pm 0.0017$ frames. 
$1 \quad$ Kumar, V. et al. Protein aggregation and neurodegenerative diseases: From theory to therapy. European Journal of Medicinal Chemistry 124, 1105-1120, doi:http://doi.org/10.1016/j.ejmech.2016.07.054 (2016).

Chiti, F. \& Dobson, C. M. Protein Misfolding, Functional Amyloid, and Human Disease. Annual Review of Biochemistry

doi:10.1146/annurev.biochem.75.101304.123901 (2006).

Pinotsi, D., Buell, A. K., Dobson, C. M., Schierle, G. S. K. \& Kaminski, C. F. A Label - Free, Quantitative Assay of Amyloid Fibril Growth Based on Intrinsic Fluorescence. ChemBioChem 14, 846-850, doi:doi:10.1002/cbic.201300103 (2013).

Nielsen, L. et al. Effect of Environmental Factors on the Kinetics of Insulin Fibril Formation: Elucidation of the Molecular Mechanism. Biochemistry 40, 6036-6046, doi:10.1021/bi002555c (2001).

Foderà, V. et al. Observation of the Early Structural Changes Leading to the Formation of Protein Superstructures. The Journal of Physical Chemistry Letters 5, 3254-3258, doi:10.1021/jz501614e (2014).

Vetri, V. \& Foderà, V. The route to protein aggregate superstructures: Particulates and amyloid-like spherulites. FEBS Letters 589, 2448-2463, doi:http://doi.org/10.1016/j.febslet.2015.07.006 (2015).

Krebs, M. R. H. et al. The formation of spherulites by amyloid fibrils of bovine insulin. Proceedings of the National Academy of Sciences of the United States of America 101, 14420-14424, doi:10.1073/pnas.0405933101 (2004).

8 Exley, C. et al. Spherulites of Amyloid-beta(42) In Vitro and in Alzheimer's Disease. Journal of Alzheimers Disease 20, 1159-1165, doi:10.3233/jad-2010-091630 (2010).

$9 \quad$ Lu, Z. P., Goh, T. T., Li, Y. \& Ng, S. C. Glass formation in La-based La-Al-Ni-Cu-(Co) alloys by Bridgman solidification and their glass forming ability. Acta Materialia 47, 2215-2224, doi:https://doi.org/10.1016/S1359-6454(99)00058-0 (1999).

10 Heaney, P. J. \& Davis, A. M. Observation and Origin of Self-Organized Textures in Agates. Science 269, 1562-1565, doi:10.1126/science.269.5230.1562 (1995).

11 Hosier, I. L., Bassett, D. C. \& Vaughan, A. S. Spherulitic Growth and Cellulation in Dilute Blends of Monodisperse Long n-Alkanes. Macromolecules 33, 8781-8790, doi:10.1021/ma000946t (2000).

12 Kajioka, H., Hikosaka, M., Taguchi, K. \& Toda, A. Branching and re-orientation of lamellar crystals in non-banded poly(butene-1) spherulites. Polymer 49, 1685-1692, doi:https://doi.org/10.1016/j.polymer.2008.01.066 (2008).

13 Jiang, Y. et al. Protein Spherulites for Sustained Release of Interferon: Preparation, Characterization and <em >in vivo</em $>$ Evaluation. Journal of Pharmaceutical Sciences 100, 1913-1922, doi:10.1002/jps.22403.

14 Krebs, M. R. H., Bromley, E. H. C., Rogers, S. S. \& Donald, A. M. The Mechanism of Amyloid Spherulite Formation by Bovine Insulin. Biophysical Journal 88, 2013-2021, doi:https://doi.org/10.1529/biophysj.104.051896 (2005).

15 Domike, K. R. \& Donald, A. M. Thermal Dependence of Thermally Induced Protein Spherulite Formation and Growth: Kinetics of $\beta$-lactoglobulin and Insulin. Biomacromolecules 8, 3930-3937, doi:10.1021/bm7009224 (2007).

16 Domike, K. R. \& Donald, A. M. Kinetics of spherulite formation and growth: Salt and protein concentration dependence on proteins $\beta$-lactoglobulin and insulin. International Journal of Biological Macromolecules

44, 301-310, doi:https://doi.org/10.1016/j.ijbiomac.2008.12.014 (2009). 
17 Toprakcioglu, Z., Challa, P., Xu, C. \& Knowles, T. P. J. Label-Free Analysis of Protein Aggregation and Phase Behavior. ACS Nano 13, 13940-13948, doi:10.1021/acsnano.9b05552 (2019).

18 Rogers, S. S., Krebs, M. R. H., Bromley, E. H. C., van der Linden, E. \& Donald, A. M. Optical Microscopy of Growing Insulin Amyloid Spherulites on Surfaces In Vitro. Biophysical Journal 90, 1043-1054, doi:10.1529/biophysj.105.072660 (2006).

19 Foderà, V., Zaccone, A., Lattuada, M. \& Donald, A. M. Electrostatics Controls the Formation of Amyloid Superstructures in Protein Aggregation. Physical Review Letters 111, 108105, doi:10.1103/PhysRevLett.111.108105 (2013).

20 Foderà, V. \& Donald, A. M. Tracking the heterogeneous distribution of amyloid spherulites and their population balance with free fibrils. The European Physical Journal E 33, 273282, doi:10.1140/epje/i2010-10665-4 (2010).

21 Huang, B., Wang, W., Bates, M. \& Zhuang, X. Three-Dimensional Super-Resolution Imaging by Stochastic Optical Reconstruction Microscopy. Science 319, 810-813, doi:10.1126/science.1153529 (2008).

22 Hayashi, S. \& Okada, Y. Ultrafast superresolution fluorescence imaging with spinning disk confocal microscope optics. Molecular Biology of the Cell 26, 1743-1751, doi:10.1091/mbc.E14-08-1287 (2015).

23 Pinholt, H. D., Bohr, S. S.-R., Iversen, J. F., Boomsma, W. \& Hatzakis, N. S. Single-particle diffusional fingerprinting: A machine-learning framework for quantitative analysis of heterogeneous diffusion. Proceedings of the National Academy of Sciences 118, e2104624118, doi:10.1073/pnas.2104624118 (2021).

24 Thomsen, J. et al. DeepFRET, a software for rapid and automated single-molecule FRET data classification using deep learning. eLife 9, doi:10.7554/eLife.60404 (2020).

25 Stella, S. et al. Conformational Activation Promotes CRISPR-Cas12a Catalysis and Resetting of the Endonuclease Activity. Cell 175, 1856-1871.e1821, doi:https://doi.org/10.1016/j.cell.2018.10.045 (2018).

26 Jensen, S. B. et al. Biased cytochrome P450-mediated metabolism via small-molecule ligands binding P450 oxidoreductase. Nature Communications 12, 2260, doi:10.1038/s41467-021-22562-w (2021).

27 Foderà, V., van de Weert, M. \& Vestergaard, B. Large-scale polymorphism and autocatalytic effect in insulin fibrillogenesis. Soft Matter 6, 4413-4419, doi:10.1039/COSM00169D (2010).

28 Foderà, V. et al. Self-Organization Pathways and Spatial Heterogeneity in Insulin Amyloid Fibril Formation. The Journal of Physical Chemistry B 113, 10830-10837, doi:10.1021/jp810972y (2009).

29 Shtukenberg, A. G., Punin, Y. O., Gunn, E. \& Kahr, B. Spherulites. Chemical Reviews 112, 1805-1838, doi:10.1021/cr200297f (2012).

30 Gránásy, L., Pusztai, T., Tegze, G., Warren, J. A. \& Douglas, J. F. Growth and form of spherulites. Physical Review E 72, 011605, doi:10.1103/PhysRevE.72.011605 (2005).

31 Shen, Y. et al. Biomolecular condensates undergo a generic shear-mediated liquid-to-solid transition. Nature Nanotechnology 15, 841-847, doi:10.1038/s41565-020-0731-4 (2020).

32 Song, S. et al. Spherulite-like Micelles. Angewandte Chemie International Edition n/a, doi:https://doi.org/10.1002/anie.202101177.

33 Galkin, O. \& Vekilov, P. G. Mechanisms of Homogeneous Nucleation of Polymers of Sickle Cell Anemia Hemoglobin in Deoxy State. Journal of Molecular Biology 336, 43-59, doi:https://doi.org/10.1016/j.jmb.2003.12.019 (2004). 
34 Moses, M. E. et al. Single-Molecule Study of Thermomyces lanuginosus Lipase in a Detergency Application System Reveals Diffusion Pattern Remodeling by Surfactants and Calcium. ACS Applied Materials \& Interfaces 13, 33704-33712, doi:10.1021/acsami.1c08809 (2021).

35 Bohr, S. S. R. et al. Direct observation of Thermomyces lanuginosus lipase diffusional states by Single Particle Tracking and their remodeling by mutations and inhibition. Sci Rep-Uk 9, 16169, doi:10.1038/s41598-019-52539-1 (2019).

36 Betzig, E. et al. Imaging Intracellular Fluorescent Proteins at Nanometer Resolution. Science 313, 1642-1645, doi:10.1126/science.1127344 (2006).

37 Ries, J. et al. Superresolution Imaging of Amyloid Fibrils with Binding-Activated Probes. ACS Chemical Neuroscience 4, 1057-1061, doi:10.1021/cn400091m (2013).

38 Cowan, N. B. \& Ivezić, Ž. The Environment of Galaxies at Low Redshift. The Astrophysical Journal 674, L13-L16, doi:10.1086/528986 (2008).

39 Goldenfeld, N. Theory of spherulitic crystallization. Journal of Crystal Growth 84, 601-608, doi:https://doi.org/10.1016/0022-0248(87)90051-0 (1987).

40 Majumder, S., Busch, H., Poudel, P., Mecking, S. \& Reiter, G. Growth Kinetics of Stacks of Lamellar Polymer Crystals. Macromolecules 51, 8738-8745, doi:10.1021/acs.macromol.8b01765 (2018).

41 Tanaka, H. \& Nishi, T. New Types of Phase Separation Behavior during the Crystallization Process in Polymer Blends with Phase Diagram. Physical Review Letters 55, 1102-1105, doi:10.1103/PhysRevLett.55.1102 (1985).

42 Buell, A. K. et al. Detailed Analysis of the Energy Barriers for Amyloid Fibril Growth. Angewandte Chemie International Edition 51, 5247-5251, doi:https://doi.org/10.1002/anie.201108040 (2012).

43 Cohen, S. I. A. et al. Distinct thermodynamic signatures of oligomer generation in the aggregation of the amyloid- $\beta$ peptide. Nat Chem 10, 523-531, doi:10.1038/s41557-0180023-x (2018).

44 Chen, W. et al. Fluorescence Self-Quenching from Reporter Dyes Informs on the Structural Properties of Amyloid Clusters Formed in Vitro and in Cells. Nano Letters 17, 143-149, doi:10.1021/acs.nanolett.6b03686 (2017).

45 Huang, B., Jones, S. A., Brandenburg, B. \& Zhuang, X. Whole-cell 3D STORM reveals interactions between cellular structures with nanometer-scale resolution. Nature Methods 5, 1047, doi:10.1038/nmeth.1274

https://www.nature.com/articles/nmeth.1274\#supplementary-information (2008).

46 Pinotsi, D. et al. Direct Observation of Heterogeneous Amyloid Fibril Growth Kinetics via Two-Color Super-Resolution Microscopy. Nano Letters 14, 339-345, doi:10.1021/nl4041093 (2014).

47 Ovesný, M., Kř́žžek, P., Borkovec, J., Švindrych, Z. \& Hagen, G. M. ThunderSTORM: a comprehensive ImageJ plug-in for PALM and STORM data analysis and super-resolution imaging. Bioinformatics 30, 2389-2390, doi:10.1093/bioinformatics/btu202 (2014).

48 Beheiry, M. E. \& Dahan, M. ViSP: representing single-particle localizations in three dimensions. Nature Methods 10, 689, doi:10.1038/nmeth.2566

https://www.nature.com/articles/nmeth.2566\#supplementary-information (2013).

49 Bates, M., Huang, B., Dempsey, G. T. \& Zhuang, X. Multicolor Super-Resolution Imaging with Photo-Switchable Fluorescent Probes. Science 317, 1749-1753, doi:10.1126/science.1146598 (2007). 
bioRxiv preprint doi: https://doi.org/10.1101/2021.08.20.457097; this version posted August 20, 2021. The copyright holder for this preprint (which was not certified by peer review) is the author/funder, who has granted bioRxiv a license to display the preprint in perpetuity. It is made available under aCC-BY-NC-ND 4.0 International license.

50 Thomsen, R. P. et al. A large size-selective DNA nanopore with sensing applications. Nat. Commun. 10, 5655, doi:10.1038/s41467-019-13284-1 (2019). 